\title{
UNIVERSITYOF BIRMINGHAM

\section{Comparing Bleeding Risk Assessment Focused on Modifiable Risk Factors Only Versus Validated Bleeding Risk Scores in Atrial Fibrillation}

\author{
Guo, Yutao; Zhu, Hang; Chen, Yundai; Lip, Gregory
}

DOI:

10.1016/j.amjmed.2017.09.009

License:

Creative Commons: Attribution-NonCommercial-NoDerivs (CC BY-NC-ND)

\section{Document Version}

Peer reviewed version

Citation for published version (Harvard):

Guo, Y, Zhu, H, Chen, Y \& Lip, G 2018, 'Comparing Bleeding Risk Assessment Focused on Modifiable Risk

Factors Only Versus Validated Bleeding Risk Scores in Atrial Fibrillation', The American Journal of Medicine, vol. 131, no. 2, pp. 185-192. https://doi.org/10.1016/j.amjmed.2017.09.009

Link to publication on Research at Birmingham portal

\section{General rights}

Unless a licence is specified above, all rights (including copyright and moral rights) in this document are retained by the authors and/or the copyright holders. The express permission of the copyright holder must be obtained for any use of this material other than for purposes permitted by law.

- Users may freely distribute the URL that is used to identify this publication.

- Users may download and/or print one copy of the publication from the University of Birmingham research portal for the purpose of private study or non-commercial research.

- User may use extracts from the document in line with the concept of 'fair dealing' under the Copyright, Designs and Patents Act 1988 (?)

- Users may not further distribute the material nor use it for the purposes of commercial gain.

Where a licence is displayed above, please note the terms and conditions of the licence govern your use of this document.

When citing, please reference the published version.

Take down policy

While the University of Birmingham exercises care and attention in making items available there are rare occasions when an item has been uploaded in error or has been deemed to be commercially or otherwise sensitive.

If you believe that this is the case for this document, please contact UBIRA@lists.bham.ac.uk providing details and we will remove access to the work immediately and investigate. 


\section{Accepted Manuscript}

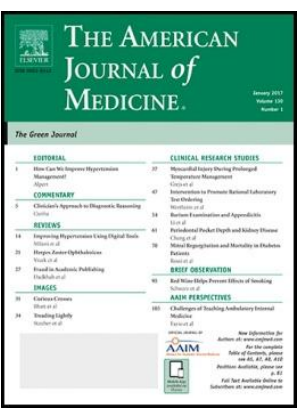

Title: Comparing Bleeding Risk Assessment Focused on Modifiable Risk

Factors Only to Validated Bleeding Risk Scores in Atrial Fibrillation

Author: Yutao Guo, Hang Zhu, Yundai Chen, Gregory Y.H. Lip

PII: $\quad$ S0002-9343(17)30938-5

DOI: $\quad$ https://doi.org/doi:10.1016/j.amjmed.2017.09.009

Reference: $\quad$ AJM 14284

To appear in: The American Journal of Medicine

Please cite this article as: Yutao Guo, Hang Zhu, Yundai Chen, Gregory Y.H. Lip, Comparing Bleeding Risk Assessment Focused on Modifiable Risk Factors Only to Validated Bleeding Risk Scores in Atrial Fibrillation, The American Journal of Medicine (2017), https://doi.org/doi:10.1016/j.amjmed.2017.09.009.

This is a PDF file of an unedited manuscript that has been accepted for publication. As a service to our customers we are providing this early version of the manuscript. The manuscript will undergo copyediting, typesetting, and review of the resulting proof before it is published in its final form. Please note that during the production process errors may be discovered which could affect the content, and all legal disclaimers that apply to the journal pertain. 
Comparing bleeding risk assessment focused on modifiable risk factors only to validated bleeding risk scores in atrial fibrillation

Yutao Guo, MD, $\mathrm{PhD}^{1}$

Hang Zhu, MD ${ }^{1}$

Yundai Chen, $\mathrm{MD}, \mathrm{PhD}^{1}$

Gregory Y H Lip, MD, ${ }^{2,3 *}$

${ }^{1}$ Department of Cardiology, Chinese PLA General Hospital, Beijing, China;

${ }^{2}$ Institute of Cardiovascular Sciences, University of Birmingham, United Kingdom;

${ }^{3}$ Thrombosis Research Unit, Department of Clinical Medicine, Aalborg University, Aalborg, Denmark

Correspondence to:

Professor Gregory Y H Lip: g.y.h.lip@bham.ac.uk

Or

Associate Professor Yutao Guo, Department of Cardiology, Chinese PLA General Hospital, Beijing, China. dor guoyt@hotmail.com

Running heading: Bleeding risk assessment in atrial fibrillation 


\section{Highlights}

- There is uncertainty whether focusing only on modifiable bleeding factors offers better bleeding risk prediction compared to validated bleeding risk scores.

- Relying on bleeding risk assessment using modifiable bleeding risk factors alone is an inferior strategy for predicting major bleeding, intracranial haemorrhage or extracranial bleeding compared to the HAS-BLED score in atrial fibrillation.

- Our observations support guideline recommendations on using the HAS-BLED score for bleeding risk assessment.

\section{ABSTRACT}

BACKGROUND There is uncertainty whether a focus on modifiable bleeding risk factors, offers better prediction of major bleeding or intracranial haemorrhage compared to other existing bleeding risk scores.

METHODS Comparison of a score based on numbers of the modifiable bleeding risk factors recommended in the 2016 European guidelines ('European risk score'), to other published bleeding risk scores that have been derived and validated in atrial fibrillation subjects (HEMORR ${ }_{2}$ HAGES, HAS-BLED, ATRIA and ORBIT) in a large hospital-based cohort of Chinese inpatients with atrial fibrillation.

Results The European score had modest predictive ability for major bleeding (cindex $0.63,95 \% \mathrm{Cl} 0.56-0.69)$ and intracranial haemorrhage $(0.72,0.65-0.79)$, but non-significantly (and poorly) predicted extracranial bleeding $(0.55,0.54-0.56$, $p=0.361$ ). The HAS-BLED score was superior to predict bleeding events compared to the European score, with the differences between c-indexes of 0.10-0.12 (Delong test, all $\mathrm{P}<0.05)$, net reclassification improvement (NRI) values of $13.0 \%-34.5 \%$ (all 
$\mathrm{p}<0.05$ ), and integrated discrimination improvement (IDI) values of $0.7 \%-1.4 \%$ (all $\mathrm{p}<0.05)$. The European score had similar predictive value to other bleeding risk schemes (HEMORR ${ }_{2} \mathrm{HAGES}, \mathrm{ATRIA}$ and ORBIT) for major bleeding and intracranial haemorrhage, as reflected by non-significant differences in c-indexes, NRI and IDI (all $p>0.05)$. HEMORR 2 HAGES and ATRIA were superior to the European score for predicting extracranial bleeding. Decision curve analysis clearly shows that HAS-BLED had better net benefit of predicting major bleeding compared to the European score.

Conclusion Relying on bleeding risk assessment using modifiable bleeding risk factors alone is an inferior strategy for predicting atrial fibrillation patients at high risk for major bleeding, intracranial haemorrhage or extracranial bleeding. Our observations re-affirm the Asian guideline recommendations on using the HAS-BLED score for bleeding risk assessment in patients with atrial fibrillation.

Key words: bleeding, risk stratification, risk factors, HAS-BLED, atrial fibrillation 


\section{INTRODUCTION}

Bleeding risk assessment is part of the overall assessment of all patients with atrial fibrillation who are started on thromboprophylaxis ${ }^{1}$. This is particularly relevant in Asia, where risks of bleeding and intracranial haemorrhage are higher in Asians compared to non-Asians on anticoagulation ${ }^{2}$.

Many risk factors for bleeding are evident but the most common ones have been used to formulate bleeding risk scores ${ }^{3}$. The latter are helpful to risk stratify patients in an objective way, aiding objective decision-making to identify those patients at high risk for bleeding, for example, where triple therapy is being considered following presentation with an acute coronary syndrome or stenting. The HAS-BLED (hypertension, abnormal renal/liver function, stroke, bleeding history or predisposition, labile INR, elderly, drugs/alcohol concomitantly) score is the most validated score in Asian subjects, and is recommended in Asian consensus/guidelines, including the 2016 Chinese Expert consensus on the management of atrial fibrillation in the elderly population and 2017 Asia-Pacific Heart Rhythm Society (APHRS) consensus guidelines on antithrombotic therapy in atrial fibrillation 456 .

Bleeding risks are also influenced by many modifiable bleeding risk factors, and attention to these is recommended in all atrial fibrillation patients who are being started on anticoagulation. Of the various scores, the HAS-BLED score was designed to 'flag up' high risk patients for regular review and follow-up, and to draw attention to the modifiable bleeding risk factors, which are contained as components of this score ${ }^{7}$. Rather than recommending a specific bleeding risk score, the 2016 European Society of Cardiology (ESC) guidelines instead directed focus to a long-tabulated list of modifiable and partially modifiable bleeding risk factors ${ }^{8}$.

User-friendly clinical assessment tools have been proposed to help the clinicians to quantify risk, to help make personalized therapeutic decisions, and not simply rely 
on guesswork when balancing risks and benefits in everyday 'real-world' practice. Various studies have investigated various modifiable, non-modifiable and biomarker-based bleeding risk factors related to atrial fibrillation ${ }^{8}$.

However, whether simply focusing on modifiable bleeding risk factors improves clinical decision-making by offering similar (or better) prediction of major bleeding or intracranial haemorrhage compared to validated bleeding risk scores has not been supported with evidence. On this basis, there is even some uncertainty whether a specific bleeding score is really needed.

We previously reported one of the largest Asian cohorts of atrial fibrillation patient where bleeding risk assessment was undertaken ${ }^{9}$. Given the relevance of bleeding risk assessment to Asian subjects, we undertook a comparison of a bleeding score based on numbers of the ESC guidelines modifiable bleeding risk factors (European risk score), to other published bleeding risk scores that have been derived and validated in atrial fibrillation subjects.

\section{METHODS}

We used the PLA General Hospital electronic health medical records database between January 1,1995 to May 30,2015 , which included the patient's medical history, therapeutic procedure, mortality data, laboratory data (Laboratory Information System, LIS), and imaging data (Picture Archiving and Communications System, PACS). The dataset has been published in detail previously ${ }^{9}$.

\section{Study population}

The consecutive patients admitted to the PLA General Hospital, Beijing, China, were identified with a primary diagnosis of atrial fibrillation or with a major co-morbid diagnosis (i.e. Secondary diagnosis) of atrial fibrillation (International Classification of Disease, Ninth Revision [ICD-9]/International Classification of Disease, tenth Revision [ICD-10] codes 427.3, 427.31/I48) between January 1,1995 to May 30, 2015. Inclusion criteria also included adult atrial fibrillation population age over 18 years, with the diagnosis of ECG or $24 \mathrm{~h}$ Holter recording. Exclusion 
criteria included patients age $<18$ years and outpatients. In total, 4824 adult patients with atrial fibrillation entered the final analysis, excluding 11 inpatients aged under 18 years and 2555 patients with readmission(s).

The study was approved by the medical ethics committee of PLA General Hospital (Approval No. S2013-064-02). There was no informed consent given this was the registry electronic health medical records study with anonymized data.

\section{Definition of Bleeding risk scores}

Bleeding risk of atrial fibrillation patients was evaluated by the available bleeding risk scores (European score for modifiable risk factors, HAS-BLED, HEMORR ${ }_{2} H A G E S$, ATRIA, and ORBIT) on admission, and the association between the bleeding risk scores and bleeding events on admission and during the in-hospital period was analyzed.

European score for modifiable risk factors: hypertension, liable INR or time in therapeutic range $<60 \%$ in patients on vitamin $k$ antagonists, medication predisposing to bleeding, such as antiplatelet drugs and non-steroidal anti-inflammatory drugs, and excess alcohol ( $\geq 8$ drinks/week), with 1 point for the presence of each risk factor. For the labile INR criterion, we recorded 'poor anticoagulation control' as assessed by the responsible physician or INR $<2.0$ at presentation.

HAS-BLED score: hypertension (uncontrolled systolic BP>160mm Hg), abnormal renal / liver function, stroke, bleeding history, labile INR, elderly (age >65 years), drugs (antiplatelets /NSAIDS) /concomitant alcohol ( $\geq 8$ units/week), with 1 point for the presence of each risk factor. Low-intermediate risk: $0-2$, high risk: $\geq 3$.

HEMORR ${ }_{2} H A G E S$ score: Hepatic or renal disease, Ethanol abuse, Malignancy, Older (aged $>75$ ), Reduced platelet count, Re-bleeding risk, uncontrolled Hypertension, Anaemia, Genetic factors (CYP 2C9 single nucleotide polymorphisms), Excessive fall risk, previous Stroke/TIA, 1 point for each risk factor present, \& 2 points for 
previous bleed. Low risk: $0-1$, intermediate risk: $2-3$, high risk: $\geq 4$. Genetic factors were not routinely measured, so this criterion scored 0 , as per previous studies.

ATRIA score: anaemia, severe renal disease (estimated glomerular filtration rate, eGFR $<30 \mathrm{ml} / \mathrm{min}$ or dialysis-dependent), age $\geq 75$ years, previous bleed, hypertension, with 1 point each for presence of previous bleed or hypertension, 2 points for age $\geq 75$, and 3 points each for presence of anaemia and renal disease. Low risk: 0 to 3, intermediate risk: 4, high risk: 5 to 10 .

ORBIT score: 1 point each for Age $>74$, insufficient kidney function (eGRF $<60$ $\mathrm{ml} / \mathrm{min} / 1.73 \mathrm{~m}^{2}$ ) and treatment with any antiplatelet, while 2 points were assigned to a positive clinical history for bleeding and the presence of anaemia or abnormal hemoglobin ( $<13 \mathrm{mg} / \mathrm{dL}$ for males and $<12 \mathrm{mg} / \mathrm{dL}$ for females). Low risk: 0 to 2 , intermediate risk: 3 , high risk: $\geq 4$.

\section{Definition of bleeding events}

As principal bleeding outcomes, we evaluated major bleeding, intracranial haemorrhage and extracranial bleeding. Major bleeding was defined according to International Society on Thrombosis and Haemostasis (ISTH) criteria, as follows: (i) fatal bleeding; and/or (ii) symptomatic bleeding in a critical area or organ (intracranial, intraspinal, intraocular, retroperitoneal, intra-articular or pericardial, or intramuscular with compartment syndrome); and/or (iii) bleeding causing a fall in hemoglobin level of $20 \mathrm{~g} / \mathrm{L}$ or more, or leading to transfusion of two or more units of whole blood or red cells ${ }^{10}$. Intracranial haemorrhage included haemorrhagic stroke, subarachnoid haemorrhage, subdural haematoma, and epidural haemorrhage, which diagnosed clinically by a neurologist and intracranial haemorrhage confirmed by computed tomography (CT) scanning or magnetic resonance imaging (MRI). Extracranial bleeding included gastrointestinal bleeding, respiratory bleeding, urinary bleeding, subcutaneous hemorrhage resulting in anemia, and fundus hemorrhage. 
Bleeding events and bleeding scores were assessed, combined ICD9/10 codes (e.g. intracranial haemorrhage, gastrointestinal bleeding, etc.), laboratory tests (e.g. a decrease in hemoglobin level of $20 \mathrm{~g} / \mathrm{L}$ or more for major bleeding; hemoglobin $<13 \mathrm{mg} / \mathrm{dL}$ for males and $<12 \mathrm{mg} / \mathrm{dL}$ for females for bleeding risk scores etc.), and medical records (e.g. transfusion of two or more units of whole blood or red cells, etc.).

Intracranial haemorrhage cases were identified by ICD-9 or ICD-10 codes $430,431,432 ; 160 . x, 161 . x$. Other major bleeding events were confirmed by [ICD-10 codes: 185.0, 198.3 K25-28 (subcodes 0-2 and 4-6 only); K62.5, K92.2, D62.9], etc. The detailed definitions of various co-morbidities and risk factors were described as the previous study, based on ICD-9 and ICD-10 codes $^{9}$.

\section{Statistical analysis.}

The predictive ability of the European score for modifiable risk factors, HAS-BLED, HEMORR ${ }_{2}$ HAGES, ATRIA, and ORBIT scores for major bleeding, intracranial haemorrhage, and extracranial bleeding were evaluated by Receptor Operating Characteristic Curve (ROC) analyses, and expressed by C-indexes (95\% confidence intervals $(\mathrm{Cl}))$.

Using the DeLong equality test, the diagnostic accuracy of the available risk scores, the differences of areas under the curve (AUC, C-statistic) of European score for modifiable risk factors were compared to other bleeding scores for significance. We also used the net reclassification improvement (NRI) and integrated discrimination improvement $(I D I)^{11}$ to further verify the predictive ability of European score for modifiable risk factors to other bleeding scores for major bleeding, intracranial haemorrhage, and extracranial bleeding. Decision curve analysis was used to quantify the net benefits related to the use of European score for modifiable risk factors, and HAS-BLED for major bleeding, respectively ${ }^{12}$.

A two-sided P-value $<0.05$ was considered as statistically significant. The $95 \%$ confidential intervals (Cls) were calculated and the statistical tests were performed 
using IBM SPSS Statistics, version 22.0 (SPSS Inc), MedCalc 12.6.1.0 (MedCalc Software), and the $\mathrm{R}$ statistical package and the on-line tutorial developed by Vickers et $a^{13}$.

\section{RESULTS}

There were 4824 patients with atrial fibrillation (mean age 67 years; 34.9\% female) during the 20-year observational period. The median (interquartile) in-hospital stay was 10(7-16) days. Of these, 481 (10\%) were on OAC, with 450 patients taking warfarin and 31 taking non-vitamin $\mathrm{K}$ antagonist coagulant (NOAC). Of the patients on warfarin, $96 \%$ had INR on admission of $<2$.

After a followup of 246 days, here were $55(1.14 \%, 95 \% \mathrm{Cl} 0.88 \%-1.48 \%)$ major bleedings, 25 (0.52\%, 95\% Cl 0.35\%-0.76\%) intracranial haemorrhage events, and $28(0.51 \%, 95 \% \mathrm{Cl} 0.38 \%-0.64 \%)$ extracranial bleeding events (gastrointestinal bleeding, respiratory bleeding, urinary bleeding, subcutaneous hemorrhage resulting in anemia, and fundus hemorrhage).

\section{Bleeding events stratified by the bleeding scores}

Major bleeding, intracranial haemorrhage, and extracranial bleeding rates (bleeds per 100, 95\% Cl) associated with bleeding risk schemes were showed in Figure 1. With increasing scores of HAS-BLED and HEMORR ${ }_{2}$ HAGES, the rates of major bleeding, intracranial haemorrhage, and extracranial bleeding significantly increased, as expected $(p<0.05)$.

\section{Predictive ability of bleeding events with different bleeding risk scores}

C-indexes for bleeding events of different bleeding risk scores ranged from 0.63-0.83 (all $p<0.05$ ) (Table 1). Of the tested scores, HAS-BLED had the highest c-indexes for major bleeding (0.72) and intracranial haemorrhage (0.83). 
The European score had modest predictive ability for major bleeding (c- index 0.63 , $95 \% \mathrm{Cl}$ 0.56-0.69) and intracranial haemorrhage $(0.72,0.65-0.79)$, but non-significantly (and poorly) predicted extracranial bleeding $(0.55,0.54-0.56$, $\mathrm{p}=0.361)$ (Table 1).

\section{Comparisons of predictive ability of European score to other bleeding scores}

The HAS-BLED score was superior to predict bleeding events compared to the European score, with the differences between c-indexes of 0.10-0.12 (Delong test, all $\mathrm{P}<0.05$ ), net reclassification improvement (NRI) values of $13.0 \%-34.5 \%$ (all $\mathrm{p}<0.05$ ), and integrated discrimination improvement (IDI) values of $0.7 \%-1.4 \%$ (all $\mathrm{p}<0.05$ ) (Table 2, Figure 2A, 2B, 2C).

The European score had similar predictive to other bleeding risk schemes (HEMORR ${ }_{2} \mathrm{HAGES}, \mathrm{ATRIA}$ and ORBIT) for major bleeding and intracranial haemorrhage, as reflected by non-significant differences in c-indexes, NRI and IDI (all $p>0.05$ ). HEMORR ${ }_{2}$ HAGES and ATRIA were superior to the European score for predicting extracranial bleeding (Table 2). Decision curve analysis showed that HAS-BLED had better net benefit of predicting major bleeding compared to the European score (Figure 3).

\section{DISCUSSION}

In this analysis, we show that relying in bleeding risk assessment using modifiable bleeding risk factors alone is an inferior strategy for predicting those atrial fibrillation patients at high risk for major bleeding, intracranial haemorrhage or extracranial bleeding. Of the established bleeding risk scores, the HAS-BLED score performed best, compared to HEMORR ${ }_{2}$ HAGES, ATRIA and ORBIT. Our observations re-affirm the the 2016 Chinese Expert consensus on the management of atrial fibrillation in the elderly population and 2017 Asia-Pacific Heart Rhythm Society (APHRS) consensus guidelines on antithrombotic therapy in atrial fibrillation ${ }^{46}$ on using the HAS-BLED score for bleeding risk assessment. 
While modifiable bleeding risk factors should be addressed in all patients with atrial fibrillation irrespective of their bleeding risk strata at every patient contact, there should be the appropriate use of a bleeding score to identify those patients at high risk for more regular review and follow-up, and to draw attention to modifiable bleeding risk factors ${ }^{14}$. This approach is important in the healthcare settings where electronic health medical records and electronic alerts are used to flag up those patients at risk. Also, clinical pathways are also driven by decision aids and protocols, and the availability of a simple practical well-validated bleeding risk score to aid objective assessment of bleeding risk that also draws attention to modifiable bleeding risk factors, such as HAS-BLED aids decision-making processes.

All the tested bleeding risk scores are clinical scores, and generally have modest predictive value for predicting outcomes ${ }^{1}$. Bleeding risk is also highly dynamic, which can be changed by addressing the modifiable bleeding risk factors that are captured within comprehensive scores such as HAS-BLED and HEMORR $\mathrm{HAGES}^{1}$. In contrast, the ATRIA and ORBIT scores would not capture some reversible bleeding risk factors ${ }^{15}$, and would therefore perform suboptimally compared to HAS-BLED, especially where warfarin is being used ${ }^{16}$. Similarly, stroke risk in atrial fibrillation can be reduced by attention to the modifiable stroke risk factors, such as uncontrolled blood pressure.

The underperformance of the European score may reflect that bleeding events are also unlikely to be solely related to modifiable bleeding risk factors. For example, an anticoagulated extremely frail 90-year-old man with atrial fibrillation and regular NSAIDs is clearly at high bleeding risk, but has only 1 modifiable bleeding risk factor; while an otherwise well 50-year-old man with regular NSAID use who is being anticoagulated for an ablation procedure would be at much lower bleeding risk, yet would be categorized as '1 modifiable bleeding risk factor'. Thus, modifiable and non-modifiable bleeding risk factors need to be considered together, as part of the holistic approach to bleeding risk assessment. 


\section{Limitations}

This study is limited by its dependence on hospital electronic health medical records but the codes have been internally validated, as per our previous study ${ }^{17}$. We also studied parameters at baseline, and determined outcomes after a observational period of 246 days, but as mentioned above, bleeding risk factors may be altered and possibly ameliorated over the follow-up duration. Nevertheless, the bleeding risk scores are at best semi-predictive and while they may aid decision-making, simple clinical scores do not catch all possible bleeding events in an individual patient. Clinicians will still need to do their own assessment of the potential risk for bleeding alongside the bleeding risk score used. Clinical judgment is still important when dealing with an individual patient at every contact, and should be exercised alongside the bleeding (or stroke) risk scores that are applied in practice, or generated by electronic health alerts.

In conclusion, bleeding risk assessment in atrial fibrillation patients only using modifiable bleeding risk factors alone is an inferior strategy for predicting those at high risk for major bleeding, intracranial haemorrhage or extracranial bleeding. Our study supports the recent Chinese consensus and Asia-Pacific Heart Rhythm Society guideline recommendations on using the HAS-BLED score for bleeding risk assessment in Asian patients with atrial fibrillation.

Acknowledgements: The study was supported by Chinese PLA Healthcare Foundation (13BJZ40), Beijing Natural Science Foundation (7142149), and National Natural Science Foundation of China (H2501). We gratefully thank Jingli Zhao, Department of Information and Technology, Chinese PLA General hospital, for data collection and processing. 
Author contributions: Y.G. and G.Y.H.L. are guarantors of the manuscript and contributed to the original idea, data analyses, and manuscript drafting and revisions. H.Z. and Y.C. contributed to the manuscript drafting and revisions. All of the authors reviewed and approved the final manuscript.

Competing financial interests: GYHL: Consultant for Bayer/Janssen, Astellas, Merck, Sanofi, BMS/Pfizer, Biotronik, Medtronic, Portola, Boelectronic health medical recordsinger Ingelheim, Microlife and Daiintracranial haemorrhagei-Sankyo. Speaker for Bayer, BMS/Pfizer, Medtronic, Boelectronic health medical recordsinger Ingelheim, Microlife, Roche and Daiintracranial haemorrhagei-Sankyo. Other authors: None declared.

Authorship: All authors had access to the data and played a role in writing this manuscript. 


\section{References}

1. Lip GY, Andreotti F, Fauchier L, et al. Bleeding risk assessment and management in atrial fibrillation patients. Executive Summary of a Position Document from the European Heart Rhythm Association [EHRA], endorsed by the European Society of Cardiology [ESC] Working Group on Thrombosis. Thrombosis and haemostasis. 2011;106(6):997-1011.

2. Chiang CE, Wang KL, Lip GY. Stroke prevention in atrial fibrillation: an Asian perspective. Thrombosis and haemostasis. 2014;111(5):789-797.

3. Zulkifly HH, Lip G, Lane D. Bleeding Risk Scores in Atrial Fibrillation and Venous Thromboembolism. Am J Cardiol. 2017 Jul 14. doi: 10.1016/j.amjcard.2017.06.058.

4. Chiang CE, Wu TJ, Ueng KC, et al. 2016 Guidelines of the Taiwan Heart Rhythm Society and the Taiwan Society of Cardiology for the management of atrial fibrillation. Journal of the Formosan Medical Association = Taiwan yi zhi. 2016;115(11):893-952.

5. Writing Committee for Expert Consensus on the Management of Atrial Fibrillation in Elderly Population, Chinese Geriatric Society, Editorial Board of Chinese Journal of Geriatrics. Expert consensus on the management of atrial fibrillation in elderly population(2016) . Chin J Geriatr. 2016,35(09): 915-928. Available at: http://zhlnyxzz.yiigle.com/CN112225201609/930117.jhtml. Accessed: August 2017.

6. Chiang CE, Okumura K, Zhang S, et al. 2017 consensus of the Asia Pacific Heart Rhythm Society on stroke prevention in atrial fibrillation. J Arrhythm. 2017;33(4):345-367.

7. Pisters R, Lane DA, Nieuwlaat R, de Vos CB, Crijns HJGM, Lip GYH. A Novel User-Friendly Score (HAS-BLED) To Assess 1-Year Risk of Major Bleeding in Patients With Atrial Fibrillation. Chest. 2010;138(5):1093-1100.

8. Kirchhof P, Benussi S, Kotecha D, et al. 2016 ESC Guidelines for the management of atrial fibrillation developed in collaboration with EACTS. European heart journal. 2016;37(38):2893-2962. 
9. Guo YT, Zhang Y, Shi XM, et al. Assessing bleeding risk in 4824 Asian patients with atrial fibrillation: The Beijing PLA Hospital Atrial Fibrillation Project. Scientific reports. 2016;6:31755.

10. Schulman S, Kearon C; Subcommittee on Control of Anticoagulation of the Scientific and Standardization Committee of the International Society on Thrombosis and Haemostasis. Definition of major bleeding in clinical investigations of antihemostatic medicinal products in non-surgical patients. J Thromb Haemost. 2005; 3(4):692-694.

11. Pencina MJ, D'Agostino RB Sr, D'Agostino RB Jr, Vasan RS. Evaluating the added predictive ability of a new marker: from area under the ROC curve to reclassification and beyond. Stat Med. 2008;27(2):157-172.

12. Vickers AJ, Elkin EB. Decision curve analysis: a novel method for evaluating prediction models. Med Decis Making. 2006;26(6):565-574.

13. Memorial Sloan Kettering Cancer Center, Decision curve analysis. New York, 2017. Available at: https:// www.mskcc.org/ departments/ epidemiology-biostatistics/health-outcomes/decision-curve-analysis-01. Accessed: August 2017.

14. Lip GY, Lane DA. Bleeding risk assessment in atrial fibrillation: observations on the use and misuse of bleeding risk scores. J Thromb Haemost. 2016;14(9):1711-1714.

15. Lip GY, Lane DA. Assessing bleeding risk in atrial fibrillation with the HAS-BLED and ORBIT scores: clinical application requires focus on the reversible bleeding risk factors. Eur Heart J. 2015;36(46):3265-3267.

16. Senoo K, Proietti M, Lane DA, Lip GY. Evaluation of the HAS-BLED, ATRIA, and ORBIT Bleeding Risk Scores in Patients with Atrial Fibrillation Taking Warfarin. Am J Med. 2016;129(6):600-607..

17. Guo Y, Tian Y, Wang H, Si Q, Wang Y, Lip GYH.Prevalence, incidence, and lifetime risk of atrial fibrillation in China: new insights into the global burden of atrial fibrillation. Chest. 2015;147(1):109-119.. 
Figure 1 Bleeding events with increasing HAS-BLED, European score, HEMORR 2 HAGES, ATRIA, and ORBIT scores.

Figure 2 Comparison of ROC curves of HAS-BLED, ATRIA, HEMORR ${ }_{2}$ HAGES, ORBIT, and European scores for major bleeding events, intracranial haemorrhage, and extracranial haemorrhage with Delong test.

2A Major bleeding events; 2B Intracranial haemorrhage; 2C Extracranial haemorrhage

Figure 3 Decision curve analysis comparing HAS-BLED and the European score for major bleeding events

This analysis shows the clinical usefulness of each score based on a continuum of potential thresholds for major bleeding ( $x$-axis) and the net benefit of using the model to stratify patients at risk ( $y$-axis) relative to assuming that no patient will have an event. 
Table 1 Predictive ability of different bleeding risk scores in 4824 Chinese patients with atrial fibrillation

\begin{tabular}{|c|c|c|c|}
\hline Major bleeding events $(n=55)$ & C index & $95 \% \mathrm{Cl}$ & $p$ \\
\hline HAS-BLED & 0.72 & $0.65-0.79$ & $<0.001$ \\
\hline $\mathrm{HEMORR}_{2} \mathrm{HAGES}$ & 0.69 & $0.62-0.77$ & $<0.001$ \\
\hline ATRIA & 0.66 & $0.58-0.74$ & $<0.001$ \\
\hline ORBIT & 0.64 & $0.56-0.73$ & $<0.001$ \\
\hline European score & 0.63 & $0.56-0.69$ & 0.001 \\
\hline Intracranial haemorrhage $(n=25)$ & C index & $95 \% \mathrm{Cl}$ & $\mathrm{p}$ \\
\hline HAS-BLED & 0.83 & $0.75-0.91$ & $<0.001$ \\
\hline $\mathrm{HEMORR}_{2} \mathrm{HAGES}$ & 0.73 & $0.61-0.85$ & $<0.001$ \\
\hline European score & 0.72 & $0.65-0.79$ & $<0.001$ \\
\hline ORBIT & 0.67 & $0.54-0.79$ & $<0.001$ \\
\hline ATRIA & 0.66 & $0.54-0.76$ & $<0.001$ \\
\hline Extracranial bleeding $(n=28)$ & Cinde & $95 \% \mathrm{Cl}$ & $\mathrm{p}$ \\
\hline ATRIA & 0.69 & $0.58-0.80$ & $<0.001$ \\
\hline $\mathrm{HEMORR}_{2} \mathrm{HAGES}$ & 0.69 & $0.68-0.71$ & 0.014 \\
\hline HAS-BLED & 0.67 & $0.66-0.68$ & 0.002 \\
\hline ORBIT & 0.65 & $0.64-0.66$ & 0.006 \\
\hline European score & 0.55 & $0.54-0.56$ & 0.361 \\
\hline
\end{tabular}

* Cl: confidential interval. Major bleeding was defined according to International Society on Thrombosis and Haemostasis (ISTH) criteria, as follows: (i) fatal bleeding; and/or (ii) symptomatic bleeding in a critical area or organ (intracranial, intraspinal, intraocular, retroperitoneal, intra-articular or pericardial, or intramuscular with compartment syndrome); and/or (iii) bleeding causing a fall in hemoglobin level of $20 \mathrm{~g} / \mathrm{L}$ or more, or leading to transfusion of two or more units of whole blood or red cells. Intracranial haemorrhage: including haemorrhagic stroke, subarachnoid haemorrhage, subdural haematoma, and epidural haemorrhage. Extracranial bleeding: including gastrointestinal bleeding, respiratory bleeding, urinary bleeding, subcutaneous hemorrhage resulting in anemia, and fundus hemorrhage. 
Table 2 Comparison of ROC curves of European score and other bleeding risk scores

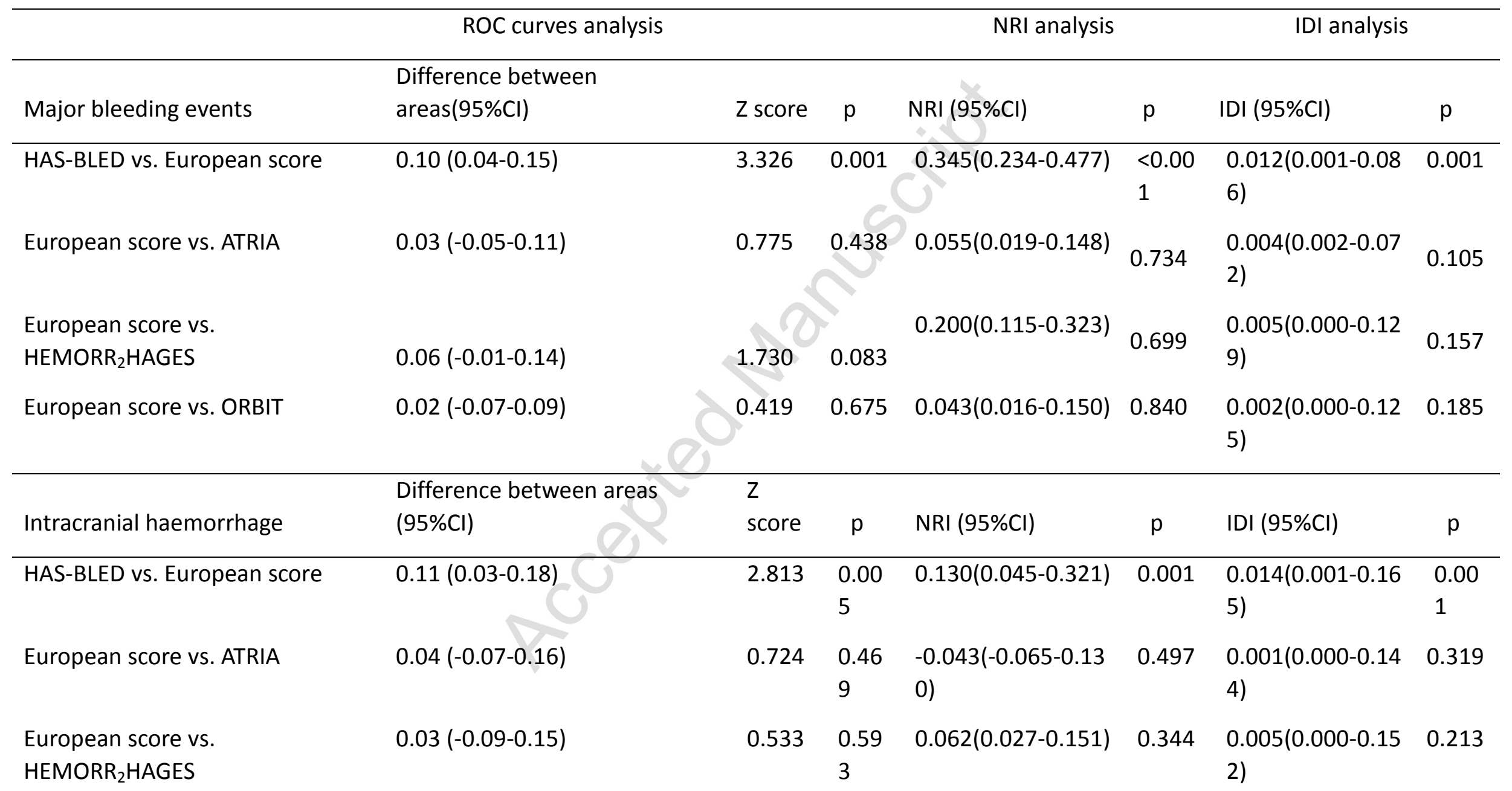




\begin{tabular}{|c|c|c|c|c|c|c|c|}
\hline European score vs. ORBIT & $0.04(-0.08-0.15)$ & 0.665 & $\begin{array}{l}0.50 \\
6\end{array}$ & $\begin{array}{l}-0.217(-0.310-0.11 \\
1)\end{array}$ & 0.714 & $\begin{array}{l}0.003(0.000-0.16 \\
9)\end{array}$ & 0.335 \\
\hline Extracranial bleeding & $\begin{array}{l}\text { Difference between areas } \\
(95 \% \mathrm{Cl})\end{array}$ & $\begin{array}{l}Z \\
\text { score }\end{array}$ & $\mathrm{p}$ & NRI $(95 \% \mathrm{Cl})$ & $p$ & IDI (95\%Cl) & $p$ \\
\hline HAS-BLED vs. European score & $0.12(0.04-0.20)$ & 3.054 & $\begin{array}{l}0.00 \\
2\end{array}$ & $0.286(0.152-0.470)$ & $\begin{array}{l}<0.00 \\
1\end{array}$ & $\begin{array}{l}0.007(0.003-0.13 \\
2)\end{array}$ & $\begin{array}{l}0.04 \\
3\end{array}$ \\
\hline $\begin{array}{l}\text { HEMORR } 2 \text { HAGES vs. European } \\
\text { score }\end{array}$ & $0.14(0.07-0.23)$ & 3.378 & $\begin{array}{l}0.00 \\
1\end{array}$ & $0.357(0.207-0.541)$ & $\begin{array}{l}<0.00 \\
1\end{array}$ & $\begin{array}{l}0.012(0.001-0.08 \\
7)\end{array}$ & $\begin{array}{l}0.00 \\
1\end{array}$ \\
\hline European score vs. ORBIT & $0.09(-0.01-0.21)$ & 1.175 & $\begin{array}{l}0.07 \\
5\end{array}$ & $\begin{array}{l}-0.143(-0.207-0.09 \\
2)\end{array}$ & 0.258 & $\begin{array}{l}0.004(0.000-0.12 \\
4)\end{array}$ & 0.139 \\
\hline
\end{tabular}

${ }^{*}$ ROC: receiver operating characteristic. NRI: net reclassification improvement. IDI: integrated discrimination improvement. 